\title{
ON THE REPRESENTATIONS OF AN ABSTRACT LATTICE AS THE FAMILY OF CLOSED SETS OF A TOPOLOGICAL SPACE
}

\author{
BY \\ DAVID DRAKE AND W. J. THRON
}

1. Introduction. In 1962 Thron considered certain questions concerning the lattice structure of the family $\mathscr{C}$ of closed sets of a topological space. In this article we continue these investigations.

Before describing the results of this paper it will be helpful to review certain definitions and give a few new ones. Whenever we talk about a topological space $(X, \mathscr{C})$ we shall mean by $\mathscr{C}$ the family of closed sets on $X$. Clearly, it defines the topological structures as well as the family of open sets, and for our purposes it is more convenient. Let $\left(\Gamma_{1}, \geqq_{1}\right)$ and $\left(\Gamma_{2}, \geqq_{2}\right)$ be two lattices; then a function $f$ from $\Gamma_{1}$ onto $\Gamma_{2}$ will be called an isomorphism iff $f$ is $1-1$ and $f$ as well as its inverse are order preserving. An isomorphism from $\Gamma$ onto itself will be called an automorphism on $\Gamma$. It is well known that $f$ is an isomorphism iff it and its inverse preserve l.u.b. and g.l.b., that is $f\left(\bigvee\left[a_{i}\right]\right)=\bigvee\left[f\left(a_{i}\right)\right]$ and $f\left(\wedge\left[a_{i}\right]\right)=\wedge\left[f\left(a_{i}\right)\right]$ and similarly for $f^{-1}$. A subset $\Delta$ of a lattice $(\Gamma, \geqq)$ is called a base of $\Gamma$ iff every element $a \in \Gamma$, other than the least element if it exists in $\Gamma$, can be written as

$$
a=\bigvee\left[d_{i}: d_{i} \in \Delta, i \in I_{a}\right] \text {. }
$$

It is convenient for our purposes to assume that if $\Gamma$ has a least element then this element does not belong to any base of $\Gamma$. Observe that we are not asserting that, for every subcollection $\Omega \subset \Delta, \vee[d: d \in \Omega]$ exists in $\Gamma$. An element $a$ of a lattice $(\Gamma, \geqq)$ will be called irreducible (strongly irreducible) iff $a$ cannot be expressed as the l.u.b. of a finite (arbitrary) number of elements of $\Gamma$, which are strictly less than $a$.

A lattice is called a set lattice iff its elements are sets and the order relation is given by set inclusion. We note that, whenever $\vee\left[C_{i}\right]$ and $\wedge\left[C_{i}\right]$ exist then

$$
\bigvee\left[C_{i}\right] \supseteq \bigcup\left[C_{i}\right] \text { and } \wedge\left[C_{i}\right] \subseteq \bigcap\left[C_{i}\right]
$$

A set lattice in which for finite index sets $I, \vee\left[C_{i}: i \in I\right]=\bigcup\left[C_{i}: i \in I\right]$ and $\wedge\left[C_{i}: i \in I\right]=\bigcap\left[C_{i}: i \in I\right]$ is called a proper set lattice. A set representation of a lattice $(\Gamma, \geqq)$ is an ordered pair $((\mathscr{C}, \supseteq), f)$, where $(\mathscr{C}, \supseteq)$ is a set lattice and $f$

Received by the editors October 9, 1964. 
is an isomorphism from $(\Gamma, \geqq)$ onto $(\mathscr{C}, \supseteq)$. A set representation is proper iff $(\mathscr{C}, \supseteq)$ is a proper set lattice. A set representation is called a topological representation iff $\mathscr{C}$ is the family of closed sets of a topological space. A (topological) representation space of a lattice $(\Gamma, \geqq)$ is an ordered pair $(X, \mathscr{C})$, where $((\mathscr{C}, \supseteq), f)$ is a (topological) representation of $(\Gamma, \geqq)$, and $X=\bigcup[C: C \in \mathscr{C}]$. A lattice $(\Gamma, \geqq)$ will be called a $\mathscr{C}$-lattice iff it is complete, distributive, and has a base consisting of irreducible elements. Thron [2] proved that a lattice has a topological representation iff it is a $\mathscr{C}$-lattice. Other characterization had previously been given by other authors. For references to these as well as to other results related to our subject the reader is referred to [2].

Thron's theorem insures that the representation families we are about to define are nonempty. A family $\mathscr{F}$ of representation spaces of a $\mathscr{C}$-lattice $(\Gamma, \geqq)$ will be called a representation family of $(\Gamma, \geqq)$ iff it satisfies the following requirements:

(i) Every $\left(X_{i}, \mathscr{C}_{i}\right) \in \mathscr{F}$ is a $T_{0}$ topological space.

(ii) If $\left(X_{i}, \mathscr{C}_{i}\right)$ and $\left(X_{j}, \mathscr{C}_{j}\right)$ are two distinct elements of $\mathscr{F}$, then the two spaces are not homeomorphic.

(iii) Every $T_{0}$ topological representation space of $(\Gamma, \geqq)$ is homeomorphic to some member of $\mathscr{F}$.

We are now in a position to describe the results of this paper. In $\S 2$ we show that every $\mathscr{C}$-lattice has a nonempty representation family, thus, incidentally providing a second proof for the sufficiency of the result of Thron, mentioned above. We then turn to the question how many distinct $T_{0}$-topological representations can a given $\mathscr{C}$-lattice have. Clearly, the restriction to $T_{0}$-spaces is necessary in order for this question to be meaningful. This question is answered in $\S 4$ in terms of the number of "occasional closures" in the given lattice. In $\S 3$ we give lattice invariant characterizations for an element of a $\mathscr{C}$-lattice to be a "necessary closure" or an "occasional closure". These concepts are central to our whole investigation. $\S 5$, finally, is devoted to a study of partial orders on a representation family. Typical results are that there always exists a greatest representation space in the family, and that if one representation space is greater than a second, then the second is homeomorphic to a dense subspace of the first.

Even though, at present, we are mainly interested in $\mathscr{C}$-lattices, we have, whenever possible, proved auxiliary theorems for more general lattices, thus throwing light on the problem of representing less restricted lattices.

It may be helpful to call attention to certain notational conventions that we shall use in this article. Abstract lattices and their subsets will be denoted by capital greek letters, while their elements will be written as lower case letters from the first part of the alphabet. For set lattices and their subsets we shall use script letters (such letters will also be used for families of other entities), while elements of set lattices will be denoted by capital letters. For the points of these sets we use letters in the latter part of the lower case alphabet. We distinguish between $\geqq$ and $>$ and similarly between $\supseteq$ and $\supset$; the second and fourth symbol 
are understood to exclude equality. By $A \sim B$ we mean the set consisting of those elements of $A$ which are not elements of $B$. The symbol thus is defined even if $B \nsubseteq A$. If there is no ambiguity as to what the ordering in a certain lattice $(\Gamma, \geqq)$ is, we frequently refer to the lattice simply as $\Gamma$. A point function $h$ from a set $X$ to a set $Y$ induces a function $H$ from the power set of $X$ to the power set of $Y$, as follows $H(A)=[h(x): x \in A]$. Since it is not likely to lead to any confusion we denote $H(A)$ by $h(A)$ so that the latter symbol is to be understood as $h(A)=[h(x): x \in A]$.

2. The existence of a representation family. In this section we shall construct topological representation spaces for a $\mathscr{C}$-lattice by using as the underlying spaces bases of irreducible elements of the lattice. In this way we obtain a representation family for every $\mathscr{C}$-lattice.

If $(\Gamma, \geqq)$ is a lattice and $\Delta$ is a base for $\Gamma$, we have for every $c \in \Gamma$

$$
c=\bigvee[d: d \in \Delta, d \leqq c]
$$

It follows that the sets

$$
\left[d: d \in \Delta, d \leqq c_{1}\right] \text { and }\left[d, d \in \Delta, d \leqq c_{2}\right]
$$

are equal iff $c_{1}=c_{2}$. For future use we define

$$
F_{c}=[d: d \in \Delta, d \leqq c] .
$$

THEOREM 2.1. Let $(\Gamma, \geqq)$ be a lattice and $\Delta$ be a subset of $\Gamma$. Then $\Delta$ is a base of $\Gamma$ iff $\left(\left(\left[F_{c}: c \in \Gamma\right], \supseteq\right), f\right)$, where $f(c)=F_{c}$, is a set representation of $(\Gamma, \geqq)$. In this case arbitrary g.l.b. in the set lattice are set intersections.

Proof. If $\Delta$ is a base then $f$ is a $1-1$ correspondence. It is easily seen that $f$ preserves order in both directions.

Now assume that $\left(\left(\left[F_{c}: c \in \Gamma\right], \supseteq\right), f\right)$ is a set representation of $\Gamma$. The family $\left[F_{c}\right]$ has a base consisting of the sets $F_{d}, d \in \Delta$, inasmuch as for every $c \in \Gamma$

$$
F_{c}=\bigcup\left[F_{d}: d \leqq c\right]=\bigvee\left[F_{d}: d \leqq c\right] .
$$

The function $f$ is an isomorphism and hence $\Delta$ is a base of $\Gamma$.

To show that g.l.b. are preserved as intersections let $c=\wedge\left[c_{i}\right]$ then $F_{c}$ $=\wedge\left[F_{c_{i}}\right] \subseteq \bigcap\left[F_{c_{i}}\right]$. Moreover if $d^{*} \in \bigcap\left[F_{c_{i}}\right]$ then $d^{*} \leqq c_{i}$ for every $i$, so that $d^{*} \leqq \bigcap\left[c_{i}\right]=c$, that is $d^{*} \in F_{c}$. It follows that $F_{c}=\bigcap\left[F_{c_{i}}\right]$.

Let $(\Gamma, \geqq)$ be a lattice and $\Delta$ a base of $\Gamma$. By the representation of $(\Gamma, \geqq)$ over $\Delta$ we shall mean the set representation defined in Theorem 2.1.

Lemma 2.1. Let $(\Gamma, \geqq)$ be a distributive lattice. Let

$$
c^{*}=\bigvee\left[c_{k}: c_{k}<c^{*}, k=1, \cdots, n\right] \text {. }
$$

If $b^{*}$ is an irreducible element of $\Gamma$ and $b^{*}<c^{*}$ then $b^{*} \leqq c_{k}$, for some $k$. 
Proof. Assume $b^{*} \leqq c_{k}$, for every $k$. Since $(\Gamma, \geqq)$ is distributive we have $b^{*}=b^{*} \wedge\left(\bigvee\left[c_{k}\right]\right)=\bigvee\left[b^{*} \wedge c_{k}\right]$. All the terms $b^{*} \wedge c_{k}$ are less than $b^{*}$, since otherwise $b^{*} \leqq c_{k}$. It follows that $b^{*}$ has a representation as the l.u.b. of a finite number of elements strictly less than $b^{*}$. This contradicts our assumption that $b^{*}$ is irreducible.

THEOREM 2.2. Let $(\Gamma$, ) be a lattice and let $\Delta$ be a base of $\Gamma$. Then the representation of $(\Gamma, \geqq)$ over $\Delta$ is proper iff $(\Gamma, \geqq)$ is distributive and $\Delta$ consists only of irreducible elements of $\Gamma$.

Proof. If the representation over $\Delta$ is proper then it is distributive and hence $\Gamma$ is distributive. Now assume that there exists a $d^{*} \in \Delta$ such that $d^{*}=\bigvee\left[c_{k}: c_{k}<d^{*}, k=1, \cdots, n\right]$. Then $F_{d^{*}}=\bigcup\left[F_{c_{k}}\right]$. This however is impossible since $d^{*} \in F_{d^{*}}$, but $d^{*} \notin F_{c_{k}}$, for any $k$. Hence every element of $\Delta$ must be irreducible.

If $\Gamma$ is distributive and $\Delta$ consists only of irreducible elements, let $c^{*}=c_{1} \vee c_{2}$, $c_{1}<c^{*}, c_{2}<c^{*}$. Then $c^{*} \notin \Delta$ and $d \in F_{c^{*}}$ implies $d<c^{*}$. It follows from Lemma 2.1 that $d<c_{1}$ or $d<c_{2}$. Hence $F_{c^{*}} \subseteq F_{c_{1}} \cup F_{c_{2}}$. On the other hand we have for all set lattices $F_{c^{*}}=F_{c_{1}} \vee F_{c_{2}} \supseteq F_{c_{1}} \cup F_{c_{2}}$. This completes the proof of the theorem since we already know from Theorem 2.1 that $\bigwedge\left[F_{c_{i}}\right]=\bigcap\left[F_{c_{i}}\right]$ for arbitrary index sets $I$, provided the g.l.b. under consideration are in the lattice.

THEOREM 2.3. Let $(\Gamma, \geqq)$ be $a \mathscr{C}$-lattice and $\Delta$ be a base of irreducible elements of $\Gamma$. Let $((\mathscr{C}, \supseteq), f)$ be the representation of $(\Gamma, \geqq)$ over $\Delta$. Then $(\Delta, \mathscr{C})$ is a $T_{0}$ topological space in which the sets $F_{d}, d \in \Delta$ are precisely the point closures.

Proof. It follows from the preceding two theorems and the fact that $\Gamma$ is a complete lattice that finite l.u.b. and arbitrary g.l.b. in $\mathscr{C}$ are finite set unions and arbitrary set intersections, respectively. Moreover, since $\Gamma$ is complete, it has a least element $l$ and a greatest element $g$, and we have

$$
F_{l}=[d: d \leqq l, d \in \Delta]=\varnothing, F_{g}=[d: d \leqq g, d \in \Delta]=\Delta .
$$

That $F_{l}=\varnothing$ follows from our convention that $l \notin \Delta$. Hence $\mathscr{C}$ is a family of closed sets on the set $\Delta$, and $(\Delta, \mathscr{C})$ is a topological space. Let $d \in \Delta$; then

$$
\overline{[d]}=\bigcap[C: d \in C, C \in \mathscr{C}]=\bigcap\left[F_{c}: d \leqq c\right]=F_{d} .
$$

Finally, since $d_{1} \neq d_{2}$ implies $F_{d_{1}} \neq F_{d_{2}}$ it follows that $(\Delta, \mathscr{C})$ is a $T_{0}$-space.

To obtain a representation family for a $\mathscr{C}$-lattice $(\Gamma, \geqq)$ we observe first that if we let $p(\Gamma)$ be the family of all bases, consisting only of irreducible elements, of $\Gamma$, then the relation $R$, defined by $\Delta_{1} R \Delta_{2}$ iff there exists an automorphism $f$ on $\Gamma$ such that $f\left(\Delta_{1}\right)=\Delta_{2}$, is an equivalence relation on $p(\Gamma)$. By $g(\Gamma)$ we shall denote a subfamily of $p(\Gamma)$ which contains exactly one element from every one of the equivalence classes into which $R$ decomposes $p(\Gamma)$. The existence of $g(\Gamma)$ 
follows from the axiom of choice in its original form. The next step in the construction is based on the following lemma.

Lemma 2.2. Let $(X, \mathscr{C})$ and $(Y, \mathscr{D})$ be two $T_{0}$ topological spaces. Then $(X, \mathscr{C})$ is homeomorphic to $(Y, \mathscr{D})$ iff there exists an isomorphism from $\mathscr{C}$ onto $\mathscr{D}$, which maps the family of point closures in $\mathscr{C}$ onto the family of point closures in $\mathscr{D}$.

Proof. Let the homeomorphism from $(X, \mathscr{C})$ to $(Y, \mathscr{D})$ be given by $h$. Since $h$ is 1-1 it induces an isomorphism from the power set of $X$ to the power set of $Y$. This isomorphism preserves closed sets in both directions since $h$ is bicontinuous. Let $h(x)=y$ then $h(\overline{[x]})$ is closed and contains $y$ so that $h(\overline{[x]}) \supset \overline{[y]}$. Similarly, $\overline{[x]} \subset h^{-1}(\overline{[y]})$, it follows that $h(\overline{[x]})=\overline{[y]}$.

Now let $f$ be the isomorphism from $\mathscr{C}$ onto $\mathscr{D}$ which maps point closures onto point closures. Then $h$ defined by $[h(x)]=f(\overline{[x]})$ is a 1-1 onto mapping from $X$ to $Y$, since both spaces are assumed to be $T_{0}$-spaces. For every closed set $C \in h(C)=f(C)$. To see this let $X \in C$ then $\overline{[x]} \subseteq C$ and hence $f(\overline{[x]})=[h(x)] \subseteq f(C)$. This establishes $h(x) \in f(C)$. If $y \in f(C)$ then $\overline{[y]} \subseteq f(C)$ so that $f^{-1}(\overline{[y]})$ $=\overline{\left[h^{-1}(y)\right]} \subseteq C$. Hence $h^{-1}(y) \in C$ and $y \in h(C)$. An analogous argument can be given for the formula $h^{-1}(D)=f^{-1}(D), D \in \mathscr{D}$. It follows that $h$ is bicontinuous.

THEOREM 2.4. Let $(\Gamma, \geqq)$ be a $\mathscr{C}$-lattice. Let $\mathscr{F}(\Gamma)=[(\Delta, \mathscr{C}): \Delta \in g(\Gamma), \mathscr{C}$ the representation of $\Gamma$ over $\Delta]$; then $\mathscr{F}$ is a representation family of $\Gamma$.

Proof. By Theorem 2.3 every space $(\Delta, \mathscr{C})$ is a $T_{0}$-space if $\Delta \in p(\Gamma)$. Hence condition (i) is satisfied. No two members of $\mathscr{F}(\Gamma)$ can be homeomorphic. This follows from Lemma 2.2. Finally, let $\left(X, \mathscr{C}_{1}\right)$ be any $T_{0}$ representation space of $(\Gamma, \geqq)$. Let the isomorphism from $\mathscr{C}_{1}$ to $\Gamma$ be given by $f$. Then $\Delta_{1}=[f(\overline{[x]}): x \in X]$ is a base of irreducible elements of $\Gamma$. To see this note that for every $C \in \mathscr{C}_{1} C$ $=\bigcup[\overline{[x]}: x \in C]$ so that the point closures form a base of $\mathscr{C}_{1}$. Further if $\overline{[x]}=\bigcup\left[C_{k}: k=1, \cdots, n, C_{k} \subset \overline{[x]}\right]$ then $x$ must be in at least one $C_{k}$ which is impossible since every $C_{k} \subset \overline{[x]}$. Hence all point closures are irreducible elements of $\mathscr{C}_{1}$. "Being irreducible" and "forming a base" are preserved under isomorphisms and hence $\Delta_{1}$ is a base of irreducible elements in $\Gamma$. It follows from the definition of $g(\Gamma)$ that there exists a $\Delta$ in $g(\Gamma)$ such that $\Delta_{1} R \Delta$. Employing Lemma 2.2 once more we can then conclude that $\left(X_{1}, \mathscr{C}_{1}\right)$ is homeomorphic to $(\Delta, \mathscr{C})$.

3. Classification of lattice elements corresponding to point closures. We shall show that the concept of point closure can be carried over from the lattice of closed sets of a topological space to more general set lattices. We are thus led to make the following definition.

Definition 3.1. Let $(\mathscr{C}, \subseteq)$ be a proper set lattice then every element of the form $\wedge[C: C \in \mathscr{C}, x \in C]$, where $x$ is a fixed element in $X=\bigcup[C: C \in \mathscr{C}]$ will be 
called a point closure in $\mathscr{C}$. Note that since the lattice may not be complete there will not, in general, be a point closure for every $x \in X$. Next, let $(\Gamma, \geqq)$ be a distributive lattice and $c \in \Gamma$. Then $c$ is called a permissible closure in $\Gamma$ iff there exists a proper set representation $((\mathscr{C}, \supseteq), f)$ of $\Gamma$ such that $f(c)$ is a point closure in $\mathscr{C}$. If $c$ is such that its image in every proper set representation of $\Gamma$ is a point closure then we shall call $c$ a necessary closure in $\Gamma$. A permissible closure which is not a necessary closure is called an occasional closure.

The reader may wonder why we have restricted ourselves in this definition to proper set representations and distributive lattices, respectively. The reason for this is that only distributive lattices can have proper set representations (and they do by a theorem due to M. H. Stone). Moreover essential use is made of the properness of the set representation in Theorem 3.1.

It may also be worthwhile to point out at this stage that not all proper representations, even for distributive lattices, need be "representations over $\Delta$ " as defined in the previous section.

THEOREM 3.1. Let $(\Gamma, \geqq)$ be a distributive lattice and let $c \in \Gamma$. Then $c$ is a permissible closure in $\Gamma$ iff $c$ is irreducible in $\Gamma$.

Proof. Assume that $c$ is a permissible closure and that it can be written as $c=\bigvee\left[c_{k}: k=1, \cdots, n\right]$. Let $((\mathscr{C}, \supseteq), f)$ be the proper representation in which $f(c)$ is a point closure. Then $f(c)=\bigcup\left[f\left(c_{k}\right)\right]$. Let $x$ be a point such that $(f c)$ is its point closure. Then $x \in f\left(c_{k}\right)$ for some $k$ and it follows that $f(c) \subseteq f\left(c_{k}\right)$, since $f(c)$ is the point closure of $x$ and thus contains all elements of $\mathscr{C}$ which contain $x$. It follows that $c \leqq c_{k}$ and that $c$ is irreducible.

If $c^{*}$ is irreducible let $(X, \mathscr{C})$ be a proper representation space of $(\Gamma, \geqq)$. If $C^{*}=f\left(c^{*}\right)$ is a point closure of $\mathscr{C}$ we have nothing more to prove. If not we form the space $\left(X^{\prime}, \mathscr{C}^{\prime}\right)$ as follows: $X^{\prime}=X \cup[X], \mathscr{C}^{\prime}$ consists of the sets $C^{\prime}$, where $C^{\prime}=C$ if $C$ q $C^{*}, C^{\prime}=C \cup[X]$ if $C \supseteq C^{*}$. The mapping $C \leftrightarrow C^{\prime}$ is easily seen to be 1-1 and order preserving in both directions, hence $\left(X^{\prime}, \mathscr{C}^{\prime}\right)$ is a representation space of $(\Gamma, \geqq)$.

To prove that $\mathscr{C}^{\prime}$ is proper we use the irreducibility of $C^{*}$ as follows. If $C \cup D \nsubseteq C^{*}$ then neither $C$ nor $D$ can, so that in that case

$$
(C \cup D)^{\prime}=C \cup D=C^{\prime} \cup D^{\prime} .
$$

If $C \cup D \supseteq C^{*}$ then $(C \cup D)^{\prime}=(C \cup D) \cup[X]$. Now $C^{*}=\left(C \cap C^{*}\right) \cup\left(D \cap C^{*}\right)$. If both $C \cap C^{*} \subset C^{*}$ and $D \cap C^{*} \subset C^{*}$ then $C^{*}$ would be reducible. It follows that either $C$ or $D$ contains $C^{*}$. If $C \supseteq C^{*}$ we have

$$
(C \cup D)^{\prime}=(C \cup D) \cup[X]=(C \cup[X]) \cup D=C^{\prime} \cup D^{\prime},
$$

and if $D \supseteq C^{*}$ we have similarly $(C \cup D)^{\prime}=C^{\prime} \cup D^{\prime}$. That $\left(C^{\prime} \cap D^{\prime}\right)=C^{\prime} \cap D^{\prime}$ can be deduced directly from the definition of the correspondence $C \leftrightarrow C^{\prime}$. 
Finally $C^{* \prime}=\wedge\left[C^{\prime}: C^{\prime} \in \mathscr{C}^{\prime},[X] \in C^{\prime}\right]$ so that $C^{* \prime}$ is a point closure in $\mathscr{C}^{\prime}$.

LEMMA 3.1. Let $c^{*}$ be an element of a lattice $(\Gamma, \geqq)$ and define $\Phi_{c^{*}}$ $=\left[c: c \in \Gamma, c<c^{*}\right]$. Then $\bigvee\left[c: c \in \Phi_{c^{*}}\right]$ exists.

Proof. The element $c^{*}$ is an upper bound of the set $\Phi_{c^{*}}$. Hence it is either its l.u.b. or there must exist another element, say $e$, in $\Gamma$ which is an upper bound of $\Phi_{c^{*}}$ and which is such that $e \geq c^{*}$. Set $b=e \wedge c^{*}$. Then it is easily seen that $b$ is the desired l.u.b. of $\Phi_{c^{*}}$.

LEMMA 3.2. Let $(\Gamma, \geqq)$ be a lattice and $\Delta$ be a base of $\Gamma$. Let $b \in \Delta$. Then $\Delta \sim[b]$ is $a$ base for $\Gamma$ iff $b=\bigvee[c: c \in \Gamma, c<b]$, that is iff $b$ is not strongly irreducible.

Proof. Suppose $b=\bigvee[c: c \in \Gamma, c<b]$. Since $\Delta$ is a base we have for every $c \in \Gamma$, $c=\bigvee\left[d: d \in F_{c}\right]$. If there exists a $c^{*}$ in $\Gamma$ such that $c^{*} \neq \bigvee\left[d: d \in F_{c^{*}} \sim[b]\right]$ then $c^{*}>b$ and the set $F_{c^{*}} \sim[b]$ has an upper bound $e \geqq c^{*}$. The element $a=e \wedge c^{*}$ then is an upper bound of the set and $a<c^{*}$. Now $b$ is the 1.u.b. of the elements $d \in \Delta, d<b$ and hence $b \geqq a$. It follows that $a \geqq \bigvee\left[d: d \in F_{c^{*}}\right]=c^{*}$ which is a contradiction.

If $b \neq \bigvee[c: c \in \Gamma, c<b]$ then $\Delta \sim[b]$ is not a base for $\Gamma$ since $b$ cannot be expressed as the l.u.b. of elements of $\Delta \sim[b]$.

THEOREM 3.2. Let $(\Gamma, \geqq)$ be a distributive lattice; then $c^{*}$ is a necessary closure in $\Gamma$ iff $c^{*}$ is strongly irreducible in $\Gamma$.

Proof. Assume $c^{*}$ is not strongly irreducible and let $(X, \mathscr{C})$ be a proper representation space of $\Gamma$. Such spaces exist. If $C^{*}$, the image of $c^{*}$ in $\mathscr{C}$, is not a point closure in $\mathscr{C}$ we have nothing further to prove. So assume that the set $P$ consisting of all those $x \in X$, for which $C^{*}$ is the point closure $x$ in $\mathscr{C}$, is not empty. Define $X^{\prime}=X \sim P$ and $\mathscr{C}^{\prime}=\left[C \cap X^{\prime}: C \in \mathscr{C}\right]$. We shall show that $\left(X^{\prime}, \mathscr{C}^{\prime}\right)$ is a proper representation space of $\Gamma$. Clearly, $\left(\mathscr{C}^{\prime}, \supseteq\right)$ is a proper set lattice since $(\mathscr{C}, \supseteq)$ is proper. Next we show that the mapping $g: C \rightarrow C^{\prime}$ is $1-1$ and that it and its inverse are order preserving. It is easily seen that $g$ itself is order preserving. To prove the other two properties of $g$ it suffices to show that $C_{1} \neq C_{2}$ implies $C_{1}^{\prime} \neq C_{2}^{\prime}$. For if $C_{1} \neq C_{2}$ then either $C_{1} \neq C_{2}$ or $C_{2} \neq C_{1}$ so that $C_{1}^{\prime} \neq C_{2}^{\prime}$. If $C_{1}^{\prime} \subseteq C_{2}^{\prime}$ then $C_{1} \subseteq C_{2}$ since $C_{2} \neq C_{1}$ implies $C_{2}^{\prime} \neq C_{1}^{\prime}$ contradicting our assumption.

Now assume that $C_{1} \notin C_{2}$; then there exist $y \in C_{2}, y \notin C_{1}$. If at least one of these $y \notin P$ then $C_{1}^{\prime} \nsubseteq C_{2}^{\prime}$ and we are through. Otherwise all $y$ are in $P$. In that case $C_{2} \subseteq C_{1} \cup P$. Moreover there exists an $x \in P \cap C_{2}$, so that since $C^{*}$ is the point closure of $x, C^{*} \subseteq C_{2}$. Also $C_{1} \cap P=\varnothing$. If this were not the case, then $P \subseteq C^{*} \subseteq C_{1}$, so that $C_{2} \subseteq C_{1} \cup P=C_{1}$, contrary to our assumption. Introduce $D=C_{1} \cap C^{*}$; then $D \subset C^{*}$ because $D=C^{*}$ implies $C_{1} \supseteq C^{*}$ which contradicts $C_{1} \cap P=\varnothing$. Let $C \subset C^{*}$; then $C \cap P=\varnothing$. Also $C \subset C^{*} \subseteq C_{2} \subseteq C_{1} \cup P$ and 
it follows that $C \subseteq C_{1}$. Hence $D$ is an upper bound for the set of all $C$ for which $C \subset C^{*}$. Thus we have $C^{*}=\bigvee\left[C: C \subset C^{*}\right] \subseteq D$, which contradicts $D \subset C^{*}$. This completes the proof that $\left(X^{\prime}, \mathscr{C}^{\prime}\right)$ is a proper representation space of $\Gamma$. In $\mathscr{C}^{\prime}$ the set $C^{* \prime}$ is not a point closure, since every point of which $C^{*}$ was a point closure has been removed from $X^{\prime}$.

If $c^{*}$ is strongly irreducible then by Lemma $3.1 c^{*}>b=\bigvee\left[c: c \in \Gamma, c<c^{*}\right]$. Then in any proper representation space $(X, \mathscr{C})$ of $\Gamma$ there exists an $x$ such that $x \in C^{*}, x \notin B$. Now assume $x \in C \in \mathscr{C}$; then $x \in C \cap C^{*}$. If $C \cap C^{*} \subset C^{*}$ then $C \cap C^{*} \subseteq B$. This is impossible since $x \in C \cap C^{*}, x \notin D$, hence $C \cap C^{*}=C^{*}$ so that $C \supset C^{*}$. But then $C^{*}$ is the point closure of $x$ in $\mathscr{C}$.

Easy consequences of the results of this section are the following corollaries.

Corollary 3.1. Let $(\Gamma, \geqq)$ be a distributive lattice; then an element $c^{*} \in \Gamma$ is an occasional closure in $\Gamma$ iff $c^{*}$ is irreducible but not strongly irreducible in $\Gamma$. An element $c^{*} \in \Gamma$ is a necessary closure in $\Gamma$ iff $c^{*}$ belongs to every base of $\Gamma$.

COROLlARY 3.2. If a lattice $(\Gamma, \geqq)$ has no base of necessary closures then every base of permissible closures of $\Gamma$ contains infinitely many occasional closures.

4. Cardinality of representation families. Trivially, the cardinality $m(\Gamma)$ of any representation family of a $\mathscr{C}$-lattice $(\Gamma, \geqq)$ is the cardinality of the family we constructed in $\S 2$. Thus $m(\Gamma)$ is equal to the number of distinct equivalence classes induced on $p(\Gamma)$, the family of all bases consisting of irreducible elements, by $R$. It thus is clear that, other things being equal, $m(\Gamma)$ becomes smaller the more automorphisms $\Gamma$ possesses, this is well illustrated in the proof of Theorem 4.2. This dependence however is hard to pin down explicitly.

More rewarding is an investigation of the dependence of $m(\Gamma)$ on $n(\Gamma)$, the number of occasional closures in $\Gamma$, and this we shall carry out here.

In the sequel we shall mean by $k(n)$ the number of cardinal numbers less than or equal to the cardinal number $n$. If $n$ is finite then $k(n)=n+1$. For $n=\aleph_{0}$ we have $k(n)=\aleph_{0}$. For $n>\aleph_{0}$ the value of $k(n)$ depends on whether the continuum hypothesis or some substitute for it is assumed. In any case $k\left(2^{\aleph_{0}}\right) \geqq \aleph_{0}$, equality holds if the continuum hypothesis is assumed. We also recall that $2^{n}$ denotes the cardinality of the power set of a set of cardinality $n$.

THEOREM 4.1. Let $n(\Gamma)$ be the number of occasional closures in a $\mathscr{C}$-lattice $(\Gamma, \geqq)$ and let $m(\Gamma)$ be the cardinality of a representation family of $(\Gamma, \geqq)$. Then

(1) $\min \left[k(n), \aleph_{0}\right] \leqq m(\Gamma) \leqq 2^{n}$,

(2) if $\Gamma$ has a base of necessary closures, $k(n) \leqq m(\Gamma) \leqq 2^{n}$.

Proof. (1) The cardinal number of distinct sets of occasional closures is $2^{n}$. This number is an upper bound for the number of distinct bases of permissible closures since, by Corollary 3.1, every base contains all of the necessary closures. Therefore $2^{n}$ is an upper bound for $m(\Gamma)$. 
Let $\Omega$ be the family of occasional closures and $\psi$ the family of necessary closures in $\Gamma$. All automorphisms preserve $\Omega$ as well as $\psi$ since the property of being an irreducible but not strongly irreducible element is preserved under all lattice isomorphisms. Let $\Delta_{1}$ and $\Delta_{2}$ be two bases of irreducible elements in $\Gamma$. If $\Delta_{1} R \Delta_{2}$ then the automorphism which maps $\Delta_{1}$ onto $\Delta_{2}$ also maps $\Omega \sim \Delta_{1}$ onto $\Omega \sim \Delta_{2}$. It follows that these two sets have the same cardinal number. Thus if two bases are such that the cardinality of $\Omega \sim \Delta_{1}$ is not equal to the cardinality of $\Omega \sim \Delta_{2}$ then $\Delta_{1}$ not $R \Delta_{2}$. Since $\Gamma$ is a $\mathscr{C}$-lattice $\Omega \cup \psi$ is a base of permissible closures and hence the sets $\psi=\left(\Omega \sim\left[a_{1}, \cdots, a_{k}\right]\right)$, where $a_{k} \in \Omega, k \leqq n$, if $n$ is finite, $k$ some natural number if $n$ is infinite, are all mutually inequivalent bases of $\Gamma$. This follows from Lemma 3.2. Thus $m(\Gamma) \geqq \min \left(k(n), \aleph_{0}\right)$.

(2) If $\psi$ is a base of $\Gamma$ then every set $\psi \cup \pi, \pi \subset \Omega$, is a base of irreducible elements of $\Gamma$. Thus if $\pi_{1}$ is cardinally inequivalent to $\pi_{2}$ then the bases $\psi \cup \pi_{1}$ and $\psi \cup \pi_{2}$ are $R$-inequivalent and hence $m(\Gamma) \geqq k(n)$ in this case.

COROLlARY 4.1. There exist, respectively, one, finitely many, or infinitely many members of a representation family of a $\mathscr{C}$-lattice $(\Gamma, \geqq)$, as there exist, respectively, zero, finitely many, or infinitely many occasional closures in $\Gamma$.

COROLlaRY 4.2. Every representation family of a $\mathscr{C}$-lattice $(\Gamma$, $\geqq)$ has exactly one element iff every irreducible element of $\Gamma$ is strongly irreducible.

We next show that the bounds $k(n)$ and $2^{n}$ for $m(\Gamma)$, obtained in Theorem 4.1 can actually be taken on for properly chosen lattices $\Gamma$.

THEOREM 4.2. For every cardinal number $n$ there exist $\mathscr{C}$-lattices $\left(\Gamma_{1}^{(n)} \geqq \geqq\right)$ and $\left(\Gamma_{2}^{(n)}, \geqq\right)$, having $n$ occasional closures, such that

$$
m\left(\Gamma_{1}^{(n)}\right)=k(n) \text { and } m\left(\Gamma_{2}^{(n)}\right)=2^{n} .
$$

Proof. Let $S^{(n)}$ be a set of cardinality $n$ and let $N$ be the set of all natural numbers. Let $X_{1}^{(n)}=S^{(n)} \cup\left(N \times S^{(n)}\right)$. Let $\Gamma_{1}^{(n)}$ consist of all subsets of $X_{1}^{(n)}$ of the forms:

(a) $A_{k, s}=\left[(m, s): m \leqq k, s \in S^{(n)}, s\right.$ fixed $]$,

(b) $A_{*, s}=\left[(m, s): m \in N, s \in S^{(n)}, s\right.$ fixed $]$,

(c) finite unions of sets of types (a) and (b),

(d) $\varnothing$ and $X_{1}^{(n)}$.

It is easily seen that $\Gamma_{1}^{(n)}$ is a family of closed sets on $X_{1}^{(n)}$ and hence is a $\mathscr{C}$-lattice. The necessary closures in $\Gamma_{1}^{(n)}$ are the sets $A_{k, s}$. The sets $A_{*, s}$ are occasional closures and so is $X_{1}^{(n)}$, if $n$ is infinite. Hence in each case there are $n$ occasional closures. The lattice $\Gamma_{1}^{(n)}$ has $n$ ! automorphism, that is to every permutation of $S^{(n)}$ there corresponds exactly one automorphisms of $\Gamma_{1}^{(n)}$. Thus two bases are $R$-inequivalent iff they are cardinally inequivalent. Since, in addition, the necessary closures form a base for $\Gamma_{1}^{(n)}$ we have $m\left(\Gamma_{1}^{(n)}\right)=k(n)$. 
To construct $\Gamma_{2}^{(n)}$ let $\left(T^{(n)}, \gg\right)$ be a well ordered set of cardinality $n$, such sets exist by the well ordering principle which is equivalent to the axiom of choice. Let $X_{2}^{(n)}=T^{(n)} \times N$. We introduce the lexicographic order on $X_{2}$, that is

$$
\left(A_{1}, k_{1}\right)>\left(A_{2}, k_{2}\right) \text { iff } A_{1}>A_{2} \text { or if } A_{1}=A_{2} \text { then } k_{1} \gg k_{2} \text {. }
$$

Now let $\Gamma_{2}^{(n)}=X_{2} \cup\left[X_{2}\right]$ and define $\left[X_{2}\right]>(A, k)$ for every $(A, k) \in X_{2}$. We thus have an ordering for $\Gamma_{2}^{(n)}$ which is linear and complete. With respect to this ordering $\Gamma_{2}^{(n)}$ can be shown to be a $\mathscr{C}$-lattice. The necessary closures are the elements $(A, k)$, $k \neq 1$. The occasional closures are the pairs $(A, 1), A \neq l$, the least element in $T^{(n)}$. The pair $(l, 1)$ is the least element in $\Gamma_{2}^{(n)}$ and thus not a point closure. $\left[X_{2}\right]$ is also a permissible closure if $n$ is infinite. Thus there are always $n$ permissible closures in $\Gamma_{2}^{(n)}$. Clearly the only automorphism of $\Gamma_{2}^{(n)}$ is the identity mapping. Thus any two distinct bases of $\Gamma_{2}^{(n)}$ are $R$-inequivalent. Since the necessary closures form a base of $\Gamma_{2}^{(n)}$ there are exactly $2^{n}$ distinct bases of irreducible elements in $\Gamma_{2}^{(n)}$ and hence $m\left(\Gamma_{2}^{(n)}\right)=2^{n}$.

Aull and Thron [1] recently introduced a new separation axiom, called the $T_{D}$-axiom, which is strictly stronger than $T_{0}$ and strictly weaker than $T_{1}$. A space satisfies the $T_{D}$-axiom iff for every $x \in X$ the set $[x]^{\prime}$, the derived set of $[x]$, is closed. Using this axiom, Thron [2] proved the theorem that, in our terminology, the subfamily of $T_{D}$-spaces in a representation family of a $\mathscr{C}$-lattice contains at most one element. This result is easily obtained from our discussion once the following theorem has been established.

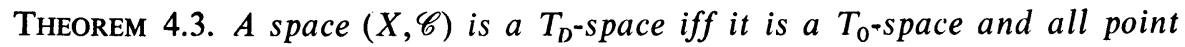
closures in the space are necessary closures.

Proof. Suppose $(X, \mathscr{C})$ is a $T_{D}$-space. For any $x$ there cannot exist a $y \neq x$ having the same closure as $x$. For if $y \in \overline{[x]}, y \neq x$ then $y \in[x]^{\prime}=\overline{[x]} \sim[x]$. The set $[x]^{\prime}$ is closed and $\left.[x]^{\prime} \neq \overline{[x}\right]$, hence $[y] \neq \overline{[x]}$ and the space is a $T_{0}$-space. Since for every $C \subset \overline{[x]}$ we have $x \notin C$ it follows that $C \subseteq[x]^{\prime}$. Thus since $[x]^{\prime}$ itself is closed $[x]^{\prime}=\bigvee[C: C \in \mathscr{C}, C \subset \overline{[x]}]$ and hence $\overline{[x]}$ is strongly irreducible, or what amounts to the same $\overline{[x]}$ is a necessary closure in $\mathscr{C}$.

If $(X, \mathscr{C})$ is a $T_{0}$-space and if the only point closures are necessary closures then for every $\overline{[x]}$ the set $D=\bigvee[C: C \in \mathscr{C}, C \subset \overline{[x]}] \subset \overline{[x]}$. Now for every $y \neq x$ and $y \in \overline{[x]}, \overline{[y]} \subset \overline{[x]}$ since the space is a $T_{0}$-space. Hence every $y \in \overline{[x]} \sim[x]$ is in $D$ and $D=[x]^{\prime}$.

It is then clear that the only $T_{D}$-spaces in a representation family are the ones for which the base of point closures corresponds to a base of necessary closures in $\Gamma$. Of these however there is at most one. The question now arises as to whether Thron's theorem could be improved, in the sense that, for some conditions on a topological space strictly weaker than $T_{D}$, the subset of a representation family meeting that condition has cardinality at most one. This can be done artificially 
for example by defining a new requirement which is either $T_{D}$ or, if the $\mathscr{C}$-lattice of the space has no $T_{D}$-respresentation, is $T_{0}$ plus the condition that all permissible closures be point closures in the space. There does not appear to be a "natural" generalization of the theorem.

A related question is whether a $\mathscr{C}$-lattice having a topological representation satisfying some well-known topological requirement, has only one member in its representation family. A sufficient condition for this is given below (compare also Thron [2, Theorem 2.3]).

THEOREM 4.4. Let $(\Gamma, \geqq)$ be a $\mathscr{C}$-lattice which has a $T_{2}$-representation space $(X, \mathscr{C})$. Then $m(\Gamma)=1$.

Proof. An equivalent condition for a space to be a $T_{2}$-space is that given $x \neq y$ there exist $C_{1}$ and $C_{2}$ in $\mathscr{C}$ such that $x \in C_{1}, x \notin C_{2}, y \in C_{2}, y \notin C_{1}$, and $C_{1} \cup C_{2}=X$. Suppose there exists an occasional closure $c^{*}$ in $\Gamma$. Then $C^{*}$ cannot be a point closure in $\mathscr{C}$ since in a $T_{2}$-space every point closure is an atom and so is a necessary closure. Therefore there exist at least two distinct points $x, y$ in $C^{*}$. There then exist sets $C_{1}$ and $C_{2}$ having the properties enumerated in the beginning of this proof. We then have $C^{*}=\left(C_{1} \cap C^{*}\right) \cup\left(C_{2} \cap C^{*}\right)$. However $y \notin C_{1} \cap C^{*}$ and $x \notin C_{2} \cap C^{*}$ so that $C_{1} \cap C^{*} \subset C^{*}$ and $C_{2} \cap C^{*} \subset C^{*}$, which contradicts the assumption that $C^{*}$ is an occasional closure, that is an irreducible element, in $\mathscr{C}$. Note that the theorem does not hold if $T_{2}$ is weakened to $T_{1}$.

We conclude this section with a result which relates the automorphisms of $\mathscr{C}$ to the autohomeomorphisms of $(X, \mathscr{C})$.

THEOREM 4.5. Let $(X, \mathscr{C})$ be a $T_{0}$-space. Then exactly those automorphisms of $\mathscr{C}$ can be extended to autohomeomorphisms on $(X, \mathscr{C})$ which leave the family of point closures of $\mathscr{C}$ invariant.

Proof. The term "extended" is used in the sense that the automorphism is induced by the homeomorphism. This result then follows easily from Lemma 2.2.

5. Partial orders on representation families. Here we introduce various order relations on a representation family, which allow us to discuss among other things whether a representation class has maximal and minimal elements. As a representation family has a certain structural resemblance to the (sufficiently restricted) family of extensions of a given topological space, so does the order introduced here have a relation to the order commonly used on extensions (or at least compactifications) of a given space. This is brought out in Theorem 5.1. Noteworthy also is the fact (see Theorem 5.2) that a greater space is an extension of the lesser space.

In the sequel it will be convenient to denote the set of point closures of a topological space $(X, \mathscr{C})$ by $\mathscr{D}(\mathscr{C})$. 
We shall say that $(X, \mathscr{C}) \geqq\left(X^{\prime}, \mathscr{C}^{\prime}\right)$ for two members of a representation family $\mathscr{F}$ of a $\mathscr{C}$-lattice $(\Gamma, \geqq)$ iff there exists an isomorphism $f$ from $\mathscr{C}^{\prime}$ onto $\mathscr{C}$ such that $f\left(\mathscr{D}\left(\mathscr{C}^{\prime}\right)\right) \subset \mathscr{D}(\mathscr{C})$. Since this is only a quasi-ordering we also introduce two other orderings on $\mathscr{F}$ which turn out to be partial orders. We define $(X, \mathscr{C}) \geqq *\left(X^{\prime}, \mathscr{C}^{\prime}\right)$ iff $(X, \mathscr{C}) \geqq\left(X^{\prime}, \mathscr{C}^{\prime}\right)$ and $\left(X^{\prime}, \mathscr{C}^{\prime}\right) \geqq(X, \mathscr{C})$. Lastly, we define $\geqq * *$ as follows: $(X, \mathscr{C}) \geqq * *\left(X^{\prime}, \mathscr{C}^{\prime}\right)$ iff for every isomorphism $f$ from $\mathscr{C}^{\prime}$ onto $\mathscr{C}$ $f\left(\mathscr{D}\left(\mathscr{C}^{\prime}\right)\right) \subset \mathscr{D}(\mathscr{C})$. It is then clear that the following inclusion relation holds:

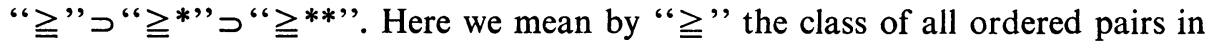
$\mathscr{F} \times \mathscr{F}$ for which the first element is $\geqq$ the second, and analogously for the other symbols.

To analyze the relation $\geqq * *$ further let us denote by $\Delta(\mathscr{C})$ the inverse image of $\mathscr{L}(\mathscr{C})$ under the isomorphism from $\Gamma$ to $\mathscr{C}$. Then $(X, \mathscr{C}) \geqq * *\left(X^{\prime}, \mathscr{C}^{\prime}\right)$ iff under every automorphism of $\Gamma$ the set $\Delta\left(\mathscr{C}^{\prime}\right)$ gets mapped into $\Delta(\mathscr{C})$. Since the identity mapping is an automorphism this means $\Delta\left(\mathscr{C}^{\prime}\right) \subset \Delta(\mathscr{C})$. It is not difficult to verify that $\geqq$, $\geqq *$, and $\geqq * *$ are reflexive and transitive and that $\geqq *$ and $\geqq * *$ are also antisymmetric. The relation $\geqq$ is not in general antisymmetric as the following example shows.

EXAMPLE 5.1 . Let $(\Gamma, \geqq)$ be the lattice consisting of $-\infty,+\infty$, all integers, and all rational numbers of the form $n+\left(2^{m}-1\right) / 2^{m}, n$ an integer and $m$ a positive integer, and let the ordering be the natural ordering. This is a $\mathscr{C}$-lattice. All nonintegral rational numbers are necessary closures and all integers and $+\infty$ are occasional closures. Therefore any set that contains the set of all necessary closures $\psi$, and any arbitrary subset of the set of occasional closures is a base of permissible closures for $\Gamma$. All automorphisms of $\Gamma$ are of the form $f_{k}$, where $f_{k}( \pm \infty)= \pm \infty$, $f_{k}(x)=x+k$ otherwise. Here $k$ can be an arbitrary integer. Now consider the two bases

$\Delta=\psi \cup[0$ and all negative integers not equal to $-(1+3 n), n \geqq 0]$,

$\Delta^{*}=\psi \cup[0$ and all negative integers not equal to $-(2+3 n), n \geqq 0]$; then $f_{-1}(\Delta) \subset \Delta^{*}$ and $f_{-2}\left(\Delta^{*}\right) \subset \Delta$. Let $\mathscr{C}$ and $\mathscr{C}^{*}$ be the representations over $\Delta$ and $\Delta^{*}$, respectively, of $\Gamma$ (see $\S 2$ ). Then we have $(\Delta, \mathscr{C}) \geqq\left(\Delta^{*}, \mathscr{C}^{*}\right)$ and $\left(\Delta^{*}, \mathscr{C}^{*}\right) \geqq(\Delta, \mathscr{C})$ and $\Delta$ not $R \Delta^{*}$ since no automorphism of $\Gamma$ can map $\Delta$ onto $\Delta^{*}$, hence $(\Delta, \mathscr{C}) \neq\left(\Delta^{*}, \mathscr{C}^{*}\right)$.

It might be of interest to try to characterize those $\mathscr{C}$-lattices $\Gamma$ for which $\geqq$ is a partial order.

We next note that the three order relations introduced here are such that if $\mathscr{F}$ and $\mathscr{G}$ are two representation families of a $\mathscr{C}$-lattice $(\Gamma, \geqq)$ and if $h$ is the mapping from $\mathscr{F}$ onto $\mathscr{G}$ which associates with every space in $\mathscr{F}$ the space in $\mathscr{G}$ which is homeomorphic to it, then $h$ is an order isomorphism with respect to all the orders considered here.

Recall that the pair $(f,(Y, \mathscr{U}))$ is an extension of a space $(X, \mathscr{T})$ iff $f$ provides a homeomorphism from $(X, \mathscr{T})$ onto a subspace $\left(S, U_{s}\right)$ of $(Y, \mathscr{U})$, and if in addition $S$ is dense in $(Y, \mathscr{U})$. Given two extensions of a space $(X, \mathscr{T})$ one defines $(f,(Y, \mathscr{U}))$ 
$\geqq(g,(Z, \mathscr{V}))$ iff there exists a continuous function $h$ from $(Y, \mathscr{U})$ onto $(Z, \mathscr{V})$ such that $h=g \circ f^{-1}$ on $f(X)$. For the ordering $\geqq$ of representation families we have the following result.

THEOREM 5.1. The relation $(X, \mathscr{C}) \geqq\left(X^{\prime}, \mathscr{C}^{\prime}\right)$ holds between two members of a representation family iff there exists a closed continuous mapping $h$ from $(X, \mathscr{C})$ onto $\left(X^{\prime}, \mathscr{C}^{\prime}\right)$ which has the additional property that for every $C \in \mathscr{C} h(C)=f(C)$, where $f$ is an isomorphism from $C$ onto $C^{\prime}$.

Proof. If $(X, \mathscr{C}) \geqq\left(X^{\prime}, \mathscr{C}^{\prime}\right)$ there exists an isomorphism $f$ from $\mathscr{C}$ to $\mathscr{C}^{\prime}$ such that $f^{-1}\left(\mathscr{D}\left(\mathscr{C}^{\prime}\right)\right) \subset \mathscr{D}(\mathscr{C})$. For every $x$ such that $\overline{[x]} \in f^{-1}\left(\mathscr{D}\left(\mathscr{C}^{\prime}\right)\right)$ define $h(x)$ so that $f(\overline{[x]})=\left[[h(x)]^{-}\right]$. For the remaining $x$ the set $f(\overline{[x]})=C^{\prime}$ is not a point closure. In this case we let $h(x)$ be some element in $C^{\prime}$. The function $h$ so defined is a mapping from $X$ onto $X^{\prime}$. Moreover, $h(C)=f(C)$ and

$$
h^{-1}\left(C^{\prime}\right)=\left[x: f(\overline{[x]}) \subset C^{\prime}\right]=\bigcup\left[\overline{[x]}: \overline{[x]} \subset f^{-1}\left(C^{\prime}\right)\right]=f^{-1}\left(C^{\prime}\right),
$$

so that $h$ is continuous. The function $h$ is closed since $h(C)=f(C)$.

Assume $h$ and $f$ are given so that for every $C \in \mathscr{C}, h(C)=f(C)$. Let $y$ be an arbitrary element in $X^{\prime}$ and set $C^{*}=h^{-1}(\overline{[y]})$ then $C^{*} \in \mathscr{C}$ since $h$ is continuous. Now suppose $C^{*}$ is not a point closure in $\mathscr{C}$; then $C^{*}=\bigcup\left[\overline{[x]}: \overline{[x]} \subset C^{*}\right]$ and

$$
\overline{[y]}=\bigcup\left[h(\overline{[x]}): \overline{[x]} \subset C^{*}\right]=\bigcup\left[f(\overline{[x]}): \overline{[x]} \subset C^{*}\right] .
$$

It follows that $y \in f(\overline{[x]}) \subset \overline{[y]}$, but then $\overline{[y]} \subset \overline{[y]}$ which is a contradiction. Hence $C^{*}=\overline{[x]}$. Note that in this argument we used the existence of the function $h$ to conclude that $\overline{[y]}=\bigcup[f(\overline{[x]})]$. Using only $f$ the best we could have concluded would have been $[y]=\bigvee[f(\overline{[x]})]$. Now let $C^{\prime}$ be an arbitrary set in $\mathscr{C}^{\prime}$; then $h^{-1}\left(C^{\prime}\right)$ is closed and hence

$$
C^{\prime}=h\left(h^{-1}\left(C^{\prime}\right)\right)=f\left(h^{-1}\left(C^{\prime}\right)\right) .
$$

Applying $f^{-1}$ to both sides of this equation we obtain $f^{-1}\left(C^{\prime}\right)=h^{-1}\left(C^{\prime}\right)$. Thus, finally, $\left.h^{-1}(\overline{[y]})=\overline{[x]}=f^{-1}(\overline{[y}]\right)$. Hence $f^{-1}\left(\mathscr{D}\left(\mathscr{C}^{\prime}\right)\right) \subset \mathscr{D}(\mathscr{C})$ and $(X, \mathscr{C}) \geqq\left(X^{\prime}, \mathscr{C}^{\prime}\right)$.

We thus see that ordering on representation families is quite closely related to ordering on extensions. However the ordering on representation families is stronger since in this case $h$ is not only continuous but also closed. It is this additional property which enables us to prove the following result for representation families (there is no corresponding result for extensions).

THEOREM 5.2. Let $(X, \mathscr{C})$ and $\left(X^{\prime}, \mathscr{C}^{\prime}\right)$ be in the same representation family and let $(X, \mathscr{C}) \geqq\left(X^{\prime}, \mathscr{C}^{\prime}\right)$. Then there exists a homeomorphism from $\left(X^{\prime}, \mathscr{C}^{\prime}\right)$ onto a dense subspace of $(X, \mathscr{C})$.

Proof. Let $f$ be an isomorphism from $\mathscr{C}$ to $\mathscr{C}^{\prime}$ which is such that 
$f^{-1}\left(\mathscr{D}\left(\mathscr{C}^{\prime}\right)\right) \subset \mathscr{D}(\mathscr{C})$ and let $D$ be the set of all $x \in X$ such that $f(\overline{[x]})=\overline{[y]}$. The function $h$ of the preceding theorem restricted to $D$ is 1-1. Hence, since it is a closed and continuous mapping it is a homeomorphism. Now let $C \in \mathscr{C}$ be such that $C \supseteq D$. Then $C \supseteq \vee\left[f^{-1}(\overline{[y]}): y \in X^{\prime}\right]$. It follows that $f(C) \supseteq \bigvee\left[\overline{[y]}: y \in X^{\prime}\right]$. The sets $\overline{[y]}$ form a base of $\mathscr{C}^{\prime}$, hence $\bigvee[\overline{[y]}]=X^{\prime}$ and $f(C)=X^{\prime}$. Thus, finally, $C=f^{-1}\left(X^{\prime}\right)=X$ and it follows that $\bar{D}=X$.

The conclusion of the theorem remains valid if $\geqq$ is replaced by $\geqq *$ or $\geqq * *$ in the assumption. This is so because $\geqq *$ implies $\geqq$ and $\geqq * *$ implies $\geqq$.

As far as the existence of greatest and least elements is concerned representation families again exhibit a better behavior than families of extensions. We have:

THEOREM 5.3. Let $\mathscr{F}$ be any representation family of a $\mathscr{C}$-lattice $(\Gamma, \geqq)$. That member $\left(X^{*}, \mathscr{C}^{*}\right)$ of $\mathscr{F}$, for which the image of the set of all point closures is the set of all permissible closures of $\Gamma$, is the greatest member of $\mathscr{F}$ with respect to $\geqq$, $*^{*}$ and $\geqq * *$.

Proof. The result is an immediate consequence of the definitions of the various order relations.

THEOREM 5.4. (1) If a $\mathscr{C}$-lattice ( $\Gamma, \geqq)$ has a base of necessary closures, then every representation family $\mathscr{F}$ of $\Gamma$ has a least member under $\geqq, \geqq *$, and $\geqq * *$. This is the space in $\mathscr{F}$ which has the property that the image of $\mathscr{D}(\mathscr{C})$ is the set of all necessary closures of $\Gamma$. (2) $A \mathscr{C}$-lattice has a base of necessary closures if a representation family has a least element under $\geqq * *$.

Proof. Part (1) follows from the fact that the set of necessary closures gets mapped onto itself by all automorphisms of $\Gamma$. To prove part (2) assume that the lattice $\Gamma$ has a least representation space $\left(X^{*}, \mathscr{C}^{*}\right)$ under $\geqq * *$, in a representation family. Assume further that the set $\mathscr{D}\left(\mathscr{C}^{*}\right)$ contain an occasional closure $C$. Then $C$ can be omitted from the base $\mathscr{D}\left(\mathscr{C}^{*}\right)$ and the remaining point closures form a base $\mathscr{D}^{\prime}$ of $\mathscr{C}^{*}$, by Lemma 3.2. But then the identity mapping on $\mathscr{C}^{*}$ fails to take $\mathscr{D}$ into $\mathscr{D}^{\prime}$ contradicting the assumption that $\left(X^{*}, \mathscr{C}^{*}\right)$ is least under $\geqq * *$. Therefore the point closures of $\mathscr{C}^{*}$ must all be necessary closures and $\mathscr{C}^{*}$ and $\Gamma$ thus have bases consisting only of necessary closures.

That part (2) of Theorem 5.3 is not valid for $\geqq *$ or $\geqq$ is seen by the following example.

EXAMPLE 5.2. The interval $[0,1]$ is a $\mathscr{C}$-lattice under its natural order, and the nonzero rationals are a base. But any base of $[0,1]$ must be a dense subset of $[0,1]$ in its usual topology. Now by a result of Cantor every dense subset of $[0,1]$ has a countable subset which is in 1-1 correspondence with the rationals under some automorphism of $[0,1]$. Let $(X, \mathscr{C})$ be a representation space of $[0,1]$ in which the point closures correspond exactly to the rationals. It follows from 
the above discussion that $(X, \mathscr{C})$ is a least element with respect to $\geqq *$ and a minimal element with respect to $\geqq$. On the other hand the interval has no necessary closures and a fortiori has no base of necessary closures.

\section{REFERENCES}

1. C. E. Aull and W. J. Thron, Separation axioms between $T_{0}$ and $T_{1}$, Indag. Math. 24 (1962), 26-37.

2. W. J. Thron, Lattice equivalence of topological spaces, Duke Math. J. 29 (1962), 671-680.

UNIVERSiTy OF BRITISH COLUMBia,

VANCOUVER, B. C., CANAda

UNIVERSITY OF COLORADO,

BOULDER, COLORADO 